\title{
Die Gegenvorschläge des Parlaments sind inakzeptabel
}

Die SVP-Volksinitiative «für tiefere Krankenkassenprämien in der Grundversicherung» hat leider in jedem der beiden eidgenössischen Räte zu einem nicht annehmbaren Gegenvorschlag geführt. Die FMH spricht sich mit aller Entschiedenheit gegen diese Vorlagen aus.

Damit eines klar ist: Die Initiative sollte dem Volk ohne Gegenvorschlag vorgelegt werden, nämlich als das, was sie ist: eine drastische Beschränkung des Zugangs zur medizinischen Versorgung für alle und ein regelrechter Angriff auf die Leistungserbringer unseres Gesundheitswesens, d.h. auf Ärzte und Pflegepersonal.

Die Gegenvorschläge der eidgenössischen Räte folgen der allgemeinen Modeströmung und versuchen, mit der Vertragsfreiheit eine alte Idee wieder aufzuwärmen, indem sie den Versicherern nichts weniger als das Recht zur Vergabe von Praxisbewilligungen einräumen wollen (Vorschlag des Ständerats). Ferner soll der Wettbewerb im Gesundheitswesen «garantiert», von unserem heutigen System mit dem Grundsatz der «optimalen Gesundheitsversorgung» zu einer «Mindestversorgung» - ein unwürdiger Rückschritt - übergegangen und schliesslich noch die «Eigenverantwortung gefördert» werden.

Die von der FMH am vergangenen Donnerstag veröffentlichte Stellungnahme verschwendet keine Zeit mit der ideologischen Tatsache, dass die Gegenvorschläge der eidgenössischen Räte im wesentlichen einem Trend folgen, sondern streicht mehrere Punkte heraus, die der Ständerat in den kommenden Wochen nicht ausser acht lassen sollte.

- Die Erteilung einer Praxisbewilligung ist ein administrativer Vorgang, der alle rechtsstaatlichen Anforderungen (Unparteilichkeit, Gleichbehandlung aller Partner, Rekursmöglichkeiten usw.) erfüllen muss und keinesfalls einer Krankenkasse überlassen werden darf.

- Es wäre völlig unangemessen, einen Grundpfeiler des Gesundheitswesens abrupt zu ändern und vom Prinzip der «optimalen Gesundheitsversorgung» zu einer «Mindestversorgung» überzugehen, wie dies im Text des Nationalrats vorgesehen ist.

Eine «optimale Gesundheitsversorgung», so wie sie zurzeit gilt, bedeutet natürlich nicht «soviel wie möglich», sondern «so gut wie möglich». Dies entspricht ganz einfach den bekannten Kriterien Wirksamkeit, Zweckmässigkeit und Wirtschaftlichkeit.

Der Wechsel zu einer Mindestversorgung wäre ein für unser Land unwürdiger Rückschritt.

Ausserdem wäre er eine unannehmbare Ungerechtigkeit für alle, die jahrelang Beiträge für eine «optimale Gesundheitsversorgung» bezahlt haben und dann, wenn sie eine Behandlung bräuchten, nur noch eine «Mindestversorgung» erhielten: ein regelrechter Betrug!

- Zwei Rechtsgutachten von renommierten Spezialisten legen völlig überzeugend dar, dass die Forderung nach
Wettbewerb im Gesundheitswesen mit jeglichem Tarifsystem unvereinbar ist. Das Kartellgesetz ist diesbezüglich klar und lässt ohne eine völlige Sinnentleerung keine Ausnahmen zu. TARMED und DRG müssten abgeschafft werden.

Wollte man im Gesundheitswesen den «Wettbewerb garantieren», müsste man das gesamte KVG neu schreiben! Vor allem aber dürfen wir nicht vergessen, dass die Tarifstrukturen neben ihrem Nutzen für die Leistungserbringer und Versicherer eine wichtige Schutzfunktion erfüllen: Sie verhindern, dass die Leistungen ausschliesslich den Regeln der Marktwirtschaft unterstehen, und bezwecken eine gerechte Verteilung der Mittel sowie die Gleichbehandlung aller. Dank der Tarifstrukturen erhalten auch Menschen mit bescheidenem Einkommen Zugang zu bezahlbaren Gesundheitsleistungen.

Kurz - und wir haben es schon oft gesagt: Die Regeln von Marktwirtschaft und Wettbewerb sind nicht auf das Gesundheitswesen anwendbar!

- Das Konzept der Eigenverantwortung geht davon aus, dass jeder seine Risikofaktoren aussuchen oder zumindest auswählen oder bewusst vermeiden kann. Wer sein Verhalten nicht anpassen will, wird hauptsächlich finanziell bestraft: keine Rückerstattung, höhere Kostenbeteiligung, steigende Prämien usw.

$\mathrm{Zu}$ den wichtigsten Risikofaktoren gehören jedoch die sogenannten sozioökonomischen Gesundheitsdeterminanten. Die typischsten davon sind Armut und Arbeitslosigkeit, und ihr Einfluss auf die Gesundheit äussert sich in den klassischen Risikofaktoren: Ernährung, Tabakkonsum, ungesunde Lebensweise, Stress usw.

Damit wird offensichtlich, dass von diesen Determinanten genau die Menschen betroffen sind, die von der Gesellschaft am meisten unterstützt und vom Staat am besten geschützt werden müssen. Eine finanzielle Bestrafung dieser Risikofaktoren würde folglich denen eine Behandlung vorenthalten, die sie am meisten bräuchten, jedoch am wenigsten finanzielle Sanktionen tragen könnten.

Der Begriff «Eigenverantwortung» ist daher in einem Gesundheitswesen, das sozial und diskriminierungsfrei sein will, fehl am Platz.

Für die FMH sind deshalb die Gegenvorschläge der eidgenössischen Räte zur SVP-Initiative klar inakzeptabel, und wir werden mit aller Macht und mit allen erforderlichen Mitteln dagegen kämpfen. Die beste Lösung wäre, sie anlässlich der kommenden Diskussion im Parlament schlicht und ergreifend fallenzulassen, weil sie eigentlich vollkommen nutzlos sind.

Dr. med. Jacques de Haller, Präsident der FMH 